For Publisher's use

\title{
ELECTRON-POSITRON- COLLIDERS
}

\author{
R.-D. HEUER \\ Institut für Experimentalphysik, Universität Hamburg, Luruper Chaussee 149, 22761 \\ Hamburg Germany \\ E-mail: rolf-dieter.heuer@desy.de
}

\begin{abstract}
An electron-positron linear collider in the energy range between 500 and $1000 \mathrm{GeV}$ is of crucial importance to precisely test the Standard Model and to explore the physics beyond it. The physics program is complementary to that of the Large Hadron Collider. Some of the main physics goals and the expected accuracies of the anticipated measurements at such a linear collider are discussed. A short review of the different collider designs presently under study is given including possible upgrade paths to the multi-TeV region. Finally a framework is presented within which the realisation of such a project could be achieved as a global international project.
\end{abstract}

\section{Introduction}

A coherent picture of matter and forces has emerged in the past decades through intensive theoretical and experimental studies. It is adequately described by the Standard Model of particle physics. In the last few years many aspects of the model have been stringently tested, some to the per-mille level, with $e^{+} e^{-}$, ep and $p \bar{p}$ machines making complementary contributions, especially to the determination of the electroweak parameters. Combining the results with neutrino scattering data and low energy measurements, the experimental analysis is in excellent concordance with the electroweak part of the Standard Model. Also the predictions of QCD have been thoroughly tested, examples being precise measurements of the strong coupling $\alpha_{s}$ and probing the proton structure to the shortest possible distances.

Despite these great successes there are many gaps in our understanding. The clearest one is the present lack of any direct evidence for the dynamics of electroweak symmetry breaking and the generation of the masses of gauge bosons and fermions. The Higgs mechanism which generates the masses of the fundamental particles in the Standard Model, has not been experimentally established though the indirect evidence from precision measurements is very strong. Even if successfully completed, the Standard Model does not provide a comprehensive theory of matter. There is no explanation for the wide range of masses of the fermions, the grand unification between the two gauge theories, electroweak and QCD, is not realised and gravity is not incorporated at the quantum level.

Several alternative scenarios have been developed for the physics which may emerge beyond the Standard Model as energies are increased. The Supersymmetric extension of the Standard Model provides a stable bridge from the presently explored energy scales up to the grand unification scale. Alternatively, new strong interactions give rise to strong forces between $W$ bosons at high energies. Quite general arguments suggest that such new phenomena must appear below a scale of approximately $3 \mathrm{TeV}$. Extra space dimensions which alter the high energy behaviour in such a way that the energy scale of gravity is in the same order as the electroweak scale are another proposed alternative.

There are two ways of exploring the new scales, through attaining the highest possible energy in a hadron collider and through high precision measurements at the energy frontier of lepton colliders.

This article is based on the results of 
many workshops on physics and detector studies for linear colliders. Much more can be found in the respective publications 1 . and on the different Web sites 6,6,8.8. Many people have contributed to these studies and the references to their work can be found in the documents quoted above.

\section{Complementarity of Lepton and Hadron Machines}

It is easier to accelerate protons to very high energies than leptons, but the detailed collision process cannot be well controlled or selected. Electron-positron colliders offer a well defined initial state. The collision energy $\sqrt{s}$ is known and it is tuneable thereby allowing the choice of the best suited centre-of-mass energy, e.g. for scanning thresholds of particle production. Furthermore, polarisation of electrons and positrons is possible. In proton collisions the rate of unwanted collision processes is very high, whereas the pointlike nature of leptons results in low backgrounds. In addition, a linear collider offers besides $e^{+} e^{-}$ collisions the options of $e^{-} e^{-}, e \gamma$ and $\gamma \gamma$ collisions which could provide important additional insight.

Hadron and Lepton Colliders are complementary and the present state of knowledge in particle physics would not have been achieved without both types of colliders running concurrently.

Telling examples from the past are internal consistency tests of the electroweak part of the Standard Model. In 1994, the precision electroweak measurements of the $Z^{0}$ boson predicted a mass of the top quark from quantum corrections of $\mathrm{M}_{\text {top }}=178 \pm 11_{-19}^{+18}$ $\mathrm{GeV}$. The direct measurement at the Tevatron in the following years yielded $\mathrm{M}_{\text {top }}=$ $174.3 \pm 5.1 \mathrm{GeV}$. The indirect measurement of $\mathrm{M}_{\mathrm{W}}=80.363 \pm 0.032 \mathrm{GeV}$ agrees well with the direct mass measurements from Tevatron and LEP of $\mathrm{M}_{\mathrm{W}}=80.450 \pm 0.039 \mathrm{GeV}$. The Standard Model has been tested and so far confirmed at the quantum level.

Much progress about the possible mass range of the Higgs boson, if it exists, has been achieved in the past around five years. Lower bounds on the mass have been derived through direct searches at LEP running with ever increasing centre-of-mass energies until the year 2000. The Standard Model Higgs contribution to electroweak observables through loop corrections provides further indirect information. Although these corrections vary only logarithmically, $\propto \log \left(M_{H} / M_{W}\right)$, the accuracy of the electroweak data obtained at LEP, SLC and the Tevatron, provides sensitivity to $M_{H}$ and in turn an upper bound for the allowed mass range. The development of these bounds is shown in figure 1. The $95 \%$ upper limit for $M_{H}$ of $196 \mathrm{GeV}$ is well within the reach of a linear collider with a centre-of-mass energy of $500 \mathrm{GeV}$.

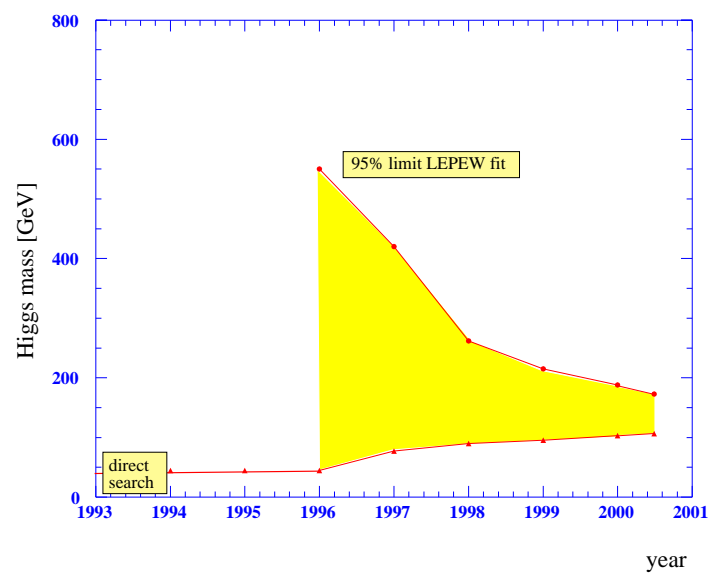

Figure 1. Development over the past years of limits for the mass of the Higgs boson from direct and indirect measurements.

In general, the physics target of the next generation of electron-positron linear colliders will be a comprehensive and high precision coverage of the energy range from $M_{Z}$ up to around $1 \mathrm{TeV}$. Energies up to around 3 to $5 \mathrm{TeV}$ could be achieved with the follow- 
ing generation of colliders. The physics case for such a machine will depend on the results from the LHC and the linear collider in the sub-TeV range.

\section{Selected Physics Topics}

In this chapter, some of the main physics topics to be studied at a linear collider will be discussed. Emphasis is given to the study of the Higgs mechanism in the Standard Model, the measurements of properties of supersymmetric particles, and precision tests of the electroweak theory. More details about these topics as well as information about the numerous topics not presented here can be found in the physics books published in the studies of the physics potential of future linear colliders .

\subsection{Standard Model Higgs Boson}

The main task of a linear electron-positron collider will be to establish experimentally the Higgs mechanism as the mechanism for generating the masses of fundamental particles:

- The Higgs boson must be discovered.

- The couplings of the Higgs boson to gauge bosons and to fermions must be proven to increase with their masses.

- The Higgs potential which generates the non-zero field in the vacuum must be reconstructed by determining the Higgs self-coupling.

- The quantum numbers $\left(J^{P C}=0^{++}\right)$ must be confirmed.

The main production mechanisms for Higgs bosons in $e^{+} e^{-}$collisions are Higgsstrahlung $e^{+} e^{-} \rightarrow H Z$ and $\mathrm{WW}$-fusion $e^{+} e^{-} \rightarrow \nu_{e} \bar{\nu}_{e} H$, and the corresponding crosssections as a function of $M_{H}$ are depicted in figure 2 for three different centre of mass energies. With an integrated luminosity of 500

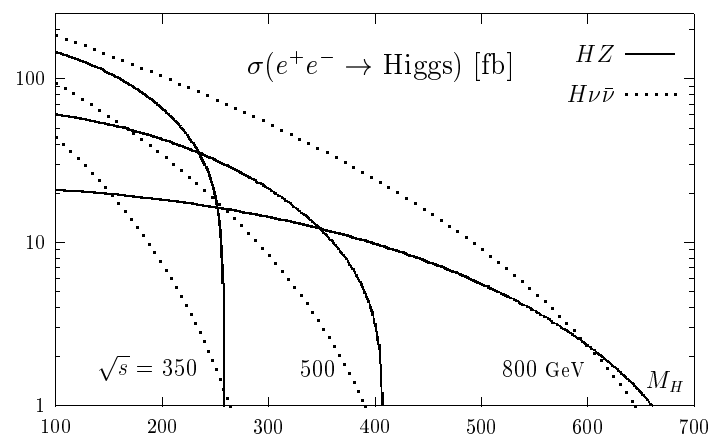

Figure 2. The Higgs-strahlung and WW fusion production cross-sections as a function of $M_{H}$ for different $\sqrt{s}$.

$f b^{-1}$, corresponding to about two years of operation, some $10^{5}$ events will be produced and can be selected with high efficiency and very low background.

The Higgs-strahlung process $e^{+} e^{-} \rightarrow$ $Z H$, with $Z \rightarrow \ell^{+} \ell^{-}$, offers a very distinctive signature ensuring the observation of the Standard Model Higgs boson up to the kinematical limit independently of its decay as illustrated in figure 3 .

The Higgs-strahlung process allows to measure the decay branching ratios of the Higgs boson and to test their dependence on the mass of the fundamental particles. The detectors proposed for linear colliders have excellent flavour tagging capability in order to distinguish the different hadronic decay modes (see for examples). Therefore, the branching ratios can be determined with accuracies of a few percent, as shown in figure 4 .

The determination of the Yukawa coupling of the Higgs boson to the top quark is provided by the process $e^{+} e^{-} \rightarrow t \bar{t} H$ at $\sqrt{s}$ of about $800 \mathrm{GeV}$; for $1000 \mathrm{fb}^{-1}$ an accuracy of $6 \%$ can be expected.

The Higgs boson quantum numbers can be determined through the rise of the cross section close to the production threshold and through the angular distributions of the $H$ and $Z$ bosons in the continuum. 


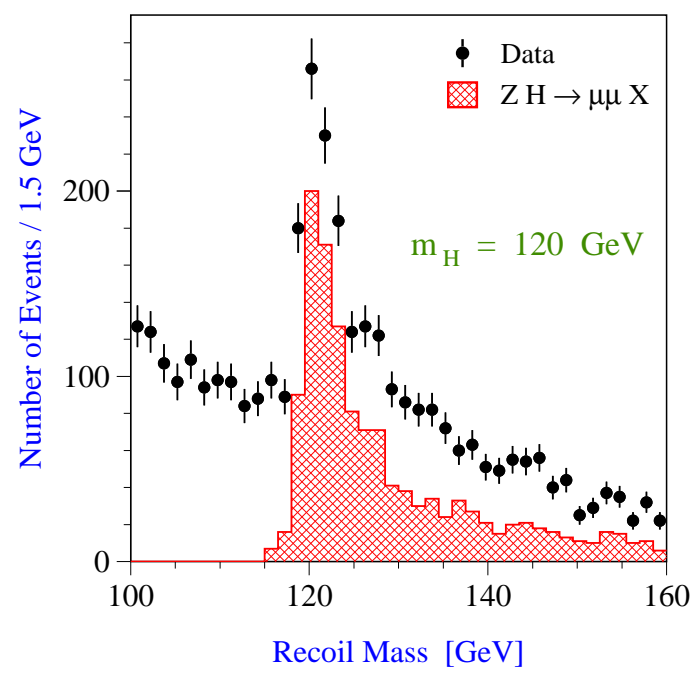

Figure 3. The $\mu^{+} \mu^{-}$recoil mass distribution in the process $e^{+} e^{-} \rightarrow H Z \rightarrow \mu^{+} \mu^{-}$for $M_{H}=120 \mathrm{GeV}$, $500 \mathrm{fb}^{-1}$ at $\sqrt{s}=350 \mathrm{GeV}$

The Higgs boson production and decay rates discussed above, can be used to determine the Higgs couplings to gauge bosons and fermions. A global fit to the measured observables optimises the available information, accounts properly for the experimental correlations between the different measurements and allows to extract the Higgs couplings in a model independent way. As an example for the accuracies reachable with the newly developed program HFITTER 4 , figure 5 shows $1 \sigma$ and $95 \%$ confidence level contours for the fitted values of the couplings $g_{c}$ and $g_{b}$ to the charm and bottom quark with comparison to the sizes of changes expected from the minimal supersymmetric extension to the Standard Model (MSSM).

To generate a non-zero value of the Higgs field in the vacuum, the minimum $\phi_{0}=v / \sqrt{2}$ of the self potential of the Higgs field $\mathrm{V}=$ $\lambda\left(\phi^{2}-\frac{1}{2} v^{2}\right)^{2}$ must be shifted away from the origin. This potential can be reconstructed by measuring the self couplings of the physical Higgs boson as predicted by the potential

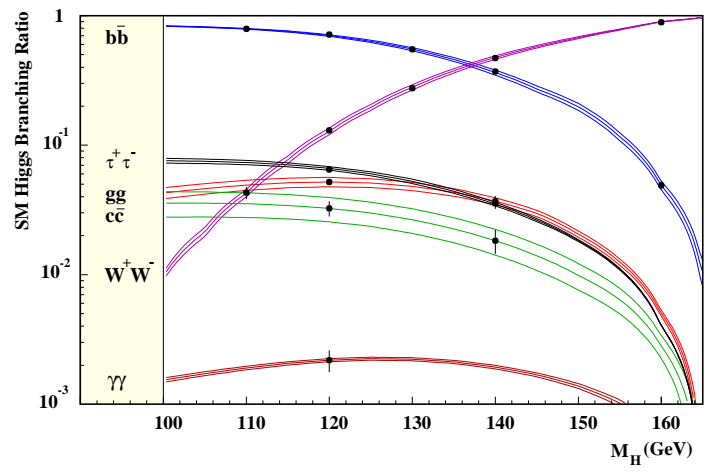

Figure 4. The predicted Standard Model Higgs boson branching ratios (bands) and the expected experimental accuracies (points with error bars).

$\mathrm{V}=\lambda v^{2} H^{2}+\lambda v H^{3}+\frac{1}{4} \lambda H^{4}$. The trilinear Higgs coupling $\lambda_{H H H}=6 \lambda v$ can be measured directly in the double Higgs-strahlung process $e^{+} e^{-} \rightarrow H H Z \rightarrow q \bar{q} b \bar{b} b \bar{b}$. The final state contains six partons resulting in a rather complicated experimental signature with six jets, a challenging task calling for excellent granularity of the tracking device and the calorimeter 6 . Despite the low cross section of the order of $0.2 \mathrm{fb}$ for $M_{H}=120 \mathrm{GeV}$ at $\sqrt{s}=500 \mathrm{GeV}$, the coupling can be measured with an accuracy of better than $20 \%$ for Higgs masses below $140 \mathrm{GeV}$ at $\sqrt{s}=500$ $\mathrm{GeV}$ with an integrated luminosity of $1 a b^{-1}$ as shown in figure 6 .

Measurements of Higgs boson properties and their anticipated accuracies are summarised in table 1 .

In summary, the Higgs mechanism can be established in an unambiguous way at a high luminosity electron-positron collider with a centre-of-mass energy up to around one $\mathrm{TeV}$ as the mechanism responsible for the spontaneous symmetry breaking of the electroweak interactions.

\subsection{Supersymmetric Particles}

Supersymmetry (SUSY) is considered the most attractive extension of the Standard 
For Publisher's use

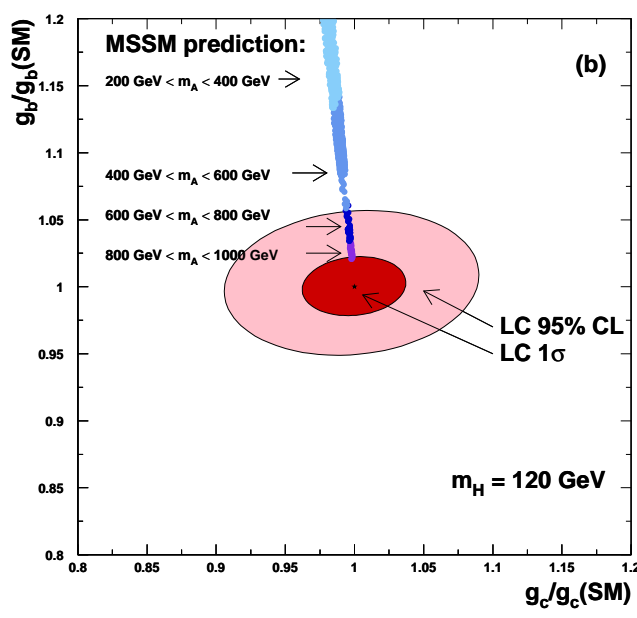

Figure 5. Higgs coupling determination: The contours for $g_{b}$ vs. $g_{c}$ for a $120 \mathrm{GeV}$ Higgs boson normalised to their Standard Model expectations as measured with $500 \mathrm{fb}^{-1}$.

Model, which cannot be the ultimate theory for many reasons. The most important feature of SUSY is that it can explain the hierarchy between the electroweak scale of $\approx 100 \mathrm{GeV}$, responsible for the $\mathrm{W}$ and $\mathrm{Z}$ masses, and the Planck scale $M_{P l} \simeq 10^{19}$ $\mathrm{GeV}$. When embedded in a grand-unified theory, it makes a very precise prediction of the electroweak mixing angle $\sin ^{2} \theta_{W}$ in excellent concordance with the precision electroweak measurement. In the following, only the minimal supersymmetric extension to the Standard Model (MSSM) will be considered and measurements of the properties of the supersymmetric particles will be discussed. Studies of the supersummetric Higgs sector can be found elsewhere国国阳.

In addition to the particles of the Standard Model, the MSSM contains their supersymmetric partners: sleptons $\tilde{l}^{ \pm}, \tilde{\nu}_{l}(l=$ $e, \mu, \tau)$, squarks $\tilde{q}$, and gauginos $\tilde{g}, \tilde{\chi}^{ \pm}, \tilde{\chi}^{0}$. In the MSSM the multiplicative quantum number R-parity is conserved, $R_{p}=+1$ for par-

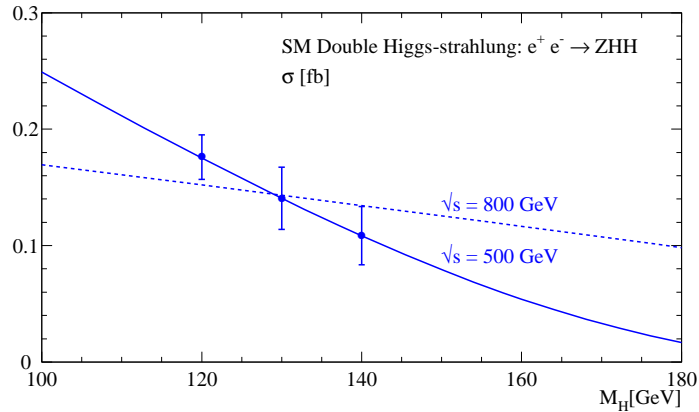

Figure 6. The cross-section for double Higgsstrahlung in the Standard Model at $\sqrt{s}$ of 500 and $800 \mathrm{GeV}$ together with the experimental accuracies expected for $1 a b^{-1}$ (points with error bars).

Table 1. Precision of the possible measurements of the Higgs boson properties.

\begin{tabular}{|l|c|c|}
\hline$m_{H}$ & $120 \mathrm{GeV}$ & $140 \mathrm{GeV}$ \\
\hline mass & $0.06 \%$ & $0.05 \%$ \\
spin & yes & yes \\
CP & yes & yes \\
\hline total width & $6 \%$ & $5 \%$ \\
\hline$g_{H Z Z}$ & $1 \%$ & $1 \%$ \\
$g_{H W W}$ & $1 \%$ & $2 \%$ \\
$g_{H b b}$ & $2 \%$ & $2 \%$ \\
$g_{H c c}$ & $3 \%$ & $10 \%$ \\
$g_{H \tau \tau}$ & $3 \%$ & $5 \%$ \\
$g_{H t t}$ & $3 \%$ & $6 \%$ \\
$\lambda_{H H H}$ & $20 \%$ & $\sim 30 \%$ \\
\hline
\end{tabular}

ticles and $R_{p}=-1$ for sparticles. Sparticles are therefore produced in pairs and they eventually decay into the lightest sparticle which has to be stable. As an example, smuons are produced and decay through the process $e^{+} e^{-} \rightarrow \tilde{\mu}^{+} \tilde{\mu}^{-} \rightarrow \mu^{+} \mu^{-} \chi_{1}^{0} \chi_{1}^{0}$ with $\chi_{1}^{0}$ as the lightest sparticle being stable and, therefore, escaping detection.

The mass scale of sparticles is only vaguely known. In most scenarios some sparticles, in particular charginos and neutralinos, are expected to lie in the energy region accessible by the next generation of $e^{+} e^{-}$ 
For Publisher's use

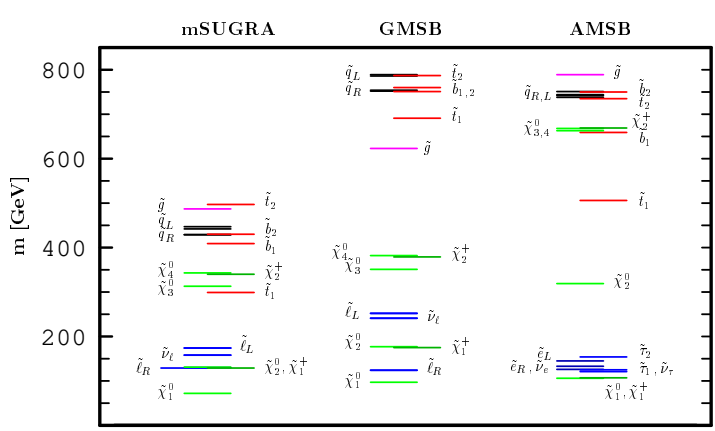

Figure 7. Examples of mass spectra in mSUGRA, GMSB and AMSB models.

colliders also supported by the recent measurement of $(g-2)_{\mu}$ 10. Examples of mass spectra for three SUSY breaking mechanisms (mSUGRA, GMSB, AMSB) are given in figure 7 .

The most fundamental problem of supersymmetric theories is how SUSY is broken and in which way this breaking is communicated to the particles. Several scenarios have been proposed in which the mass spectra are generally quite different as illustrated in figure 7. High precision measurements of the particle properties are therefore expected to distinguish between some of these scenarios. The study and exploration of Supersymmetry will proceed in the following steps:

- Reconstruction of the kinematically accessible spectrum of sparticles and the measurement of their properties, masses and quantum numbers

- Extraction of the basic low-energy parameters such as mass parameters, couplings, and mixings

- Analysis of the breaking mechanism and reconstruction of the underlying theory.

While it is unlikely that the complete spectrum of sparticles will be accessible at a collider with $\sqrt{s}$ up to around $1 \mathrm{TeV}$, a signifi- cant part of the spectrum should be measureable. In general, at an $e^{+} e^{-}$collider production cross sections are large and backgrounds are rather small. Masses of sparticles can be determined from the decay kinematics, measured in the continuum. An example for such mesurements is given in figure 8. Typical accuracies are of the order 100 to $300 \mathrm{MeV}$. Excellent mass resolutions of the order of 50 $\mathrm{MeV}$ with an integrated luminosity of 100 $f b^{-1}$ can be obtained for the light charginos and neutralinos through the measurement of the excitation curves at production threshold, as also shown in figure 9 .
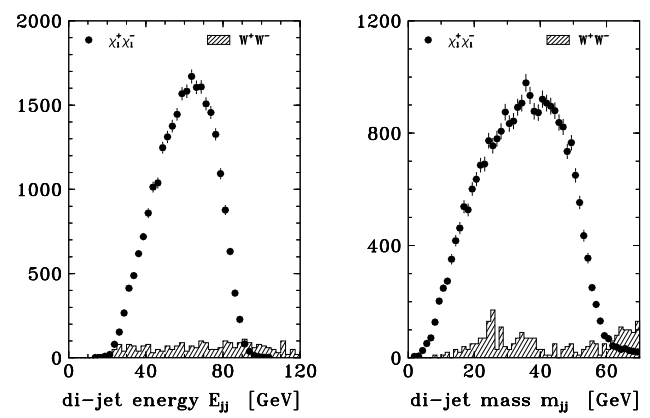

Figure 8. Di-jet energy and mass spectra at $\sqrt{s}=320$ $\mathrm{GeV}$ and $160 \mathrm{fb}^{-1}$ for the reaction $e^{+} e^{-} \rightarrow \tilde{\chi}_{1}^{+} \tilde{\chi}_{1}^{-} \rightarrow$ $\ell^{ \pm} \nu \tilde{\chi}_{1}^{0} q \bar{q}^{\prime} \tilde{\chi}_{1}^{0}$.

The reconstruction of the mechanism which breaks supersymmetry will give significant insight into the laws of Nature at energy scales where gravity becomes important as quantum effect. Various models like minimal supergravity mSURGA), gauge mediated SUSY breaking (GMSB), or anomaly mediated SUSY breaking (AMSB) have been proposed. These mechanisms lead to different spectra of sparticle masses as was shown already in figure 7 . The supersymmetric renormalisation group equations (RGE's) are largely independent of the assumed properties of the specific SUSY model at high energies. This can be used to interpret the measured SUSY spectra. In a 'bottom up' 
For Publisher's use

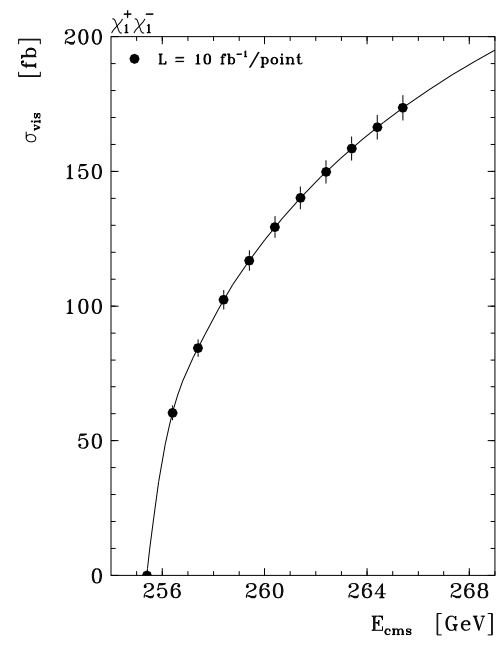

Figure 9. Cross section near threshold for the process $e^{+} e^{-} \rightarrow \tilde{\chi}_{1}^{+} \tilde{\chi}_{1}^{-}, 10 \mathrm{fb}^{-1}$ per point.

approach, the measured electroweak scale SUSY parameters are extrapolated to high energies using these RGE's.

Due to the high precision of the measured input variables, only possible at the linear collider, an accurate test can be performed at which energy scale certain parameters become equal. Most interesting, the assumption of grand unification of forces requires the gaugino mass parameters $M_{1}, M_{2}, M_{3}$ to meet at the GUT scale (figure 10 (left)). Different SUSY breaking mechanisms predict different unification patterns of the sfermion mass parameters at high energy. With the high accuracy of the linear collider measurements these models can be distinguished as shown in figure 10 for the case of mSUGRA (middle) and GMSB (right).

In summary, the high precision studies of supersymmetric particles and their properties can open a window to energy scales far above the scales reachable with future accelerators, possibly towards the Planck scale where gravity becomes important.

\subsection{Precision Measurements}

The primary goal of precision measurements of gauge boson properties is to establish the non-abelian nature of electroweak interactions. The gauge symmetries of the Standard Model determine the form and the strength of the self-interactions of the electroweak bosons, the triple couplings $W W \gamma$ and $W W Z$ and the quartic couplings. Deviations from the Standard Model expectations for these couplings could be expected in several scenarios, for example in models where there exists no light Higgs boson and where the $W$ and $Z$ bosons are generated dynamically and interact strongly at high scales. Also for the extrapolation of couplings to high scales to test theories of grand unification such high precision measurements are mandatory. For the study of the couplings between gauge bosons the best precision is reached at the highest possible centre of mass energies. These couplings are especially sensitive to models of strong electroweak symmetry breaking.

$\mathrm{W}$ bosons are produced either in pairs, $e^{+} e^{-} \rightarrow W^{+} W^{-}$or singly, $e^{+} e^{-} \rightarrow W e \nu$ with both processes being sensitive to the triple gauge couplings. In general the total errors estimated on the anomalous couplings are in the range of few $\times 10^{-4}$. Figure 11 compares the precision obtainable for $\Delta \kappa_{\gamma}$ and $\Delta \lambda_{\gamma}$ at different machines.

The measurements at a linear collider are sensitive to strong symmetry breaking beyond $\Lambda$ of the order of $5 \mathrm{TeV}$, to be compared with the electroweak symmetry breaking scale $\Lambda_{E W S B}=4 \pi v \approx 3 \mathrm{TeV}$.

One of the most sensitive quantities to loop corrections from the Higgs boson is the effective weak mixing angle in $Z$ boson decays. By operating the collider at energies close to the $Z$-pole with high luminosity (GigaZ) to collect at least $10^{9} Z$ bosons in particular the accuracy of the measure- 

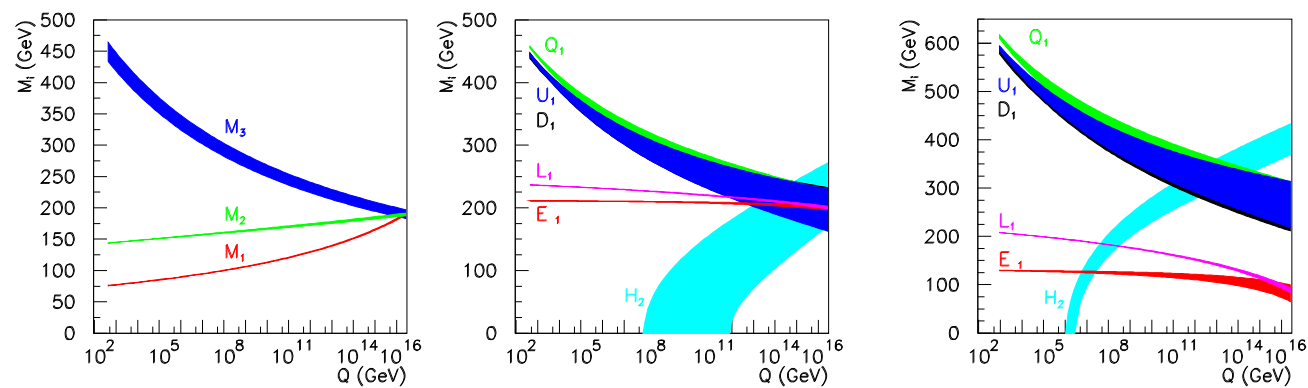

Figure 10. Extrapolation of SUSY parameters measured at the electroweak scale to high energies.
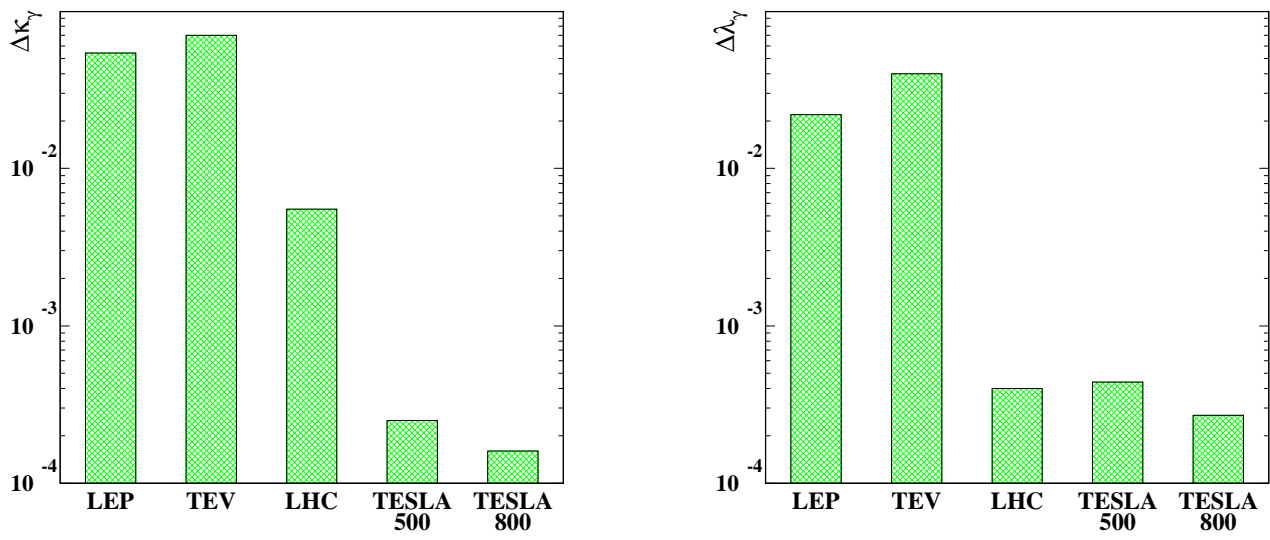

Figure 11. Comparison of constraints on the anomalous couplings $\Delta \kappa_{\gamma}$ and $\Delta \lambda_{\gamma}$ at different machines

ment of $\sin ^{2} \theta_{\text {eff }}^{l}$ can be improved by one order of magnitude wrt. the precision obtained today 11 . With both electron and positron beams longitudinally polarised, $\sin ^{2} \theta_{\text {eff }}^{l}$ can be determined most accurately by measuring the left-right asymmetry $A_{L R}=A_{e}=$ $2 v_{e} a_{e} /\left(v_{e}^{2}+a_{e}^{2}\right)$ with $v_{e}\left(a_{e}\right)$ being the vector (axialvector) couplings of the $Z$ boson to the electron and $v_{e} / a_{e}=1-4 \sin ^{2} \theta_{\text {eff }}^{l}$ for pure $Z$ exchange. Particularly demanding is the precision of $2 \times 10^{-4}$ with which the polarisation needs to be known to match the statistical accuracy. An error in the weak mixing angle of $\Delta \sin ^{2} \theta_{\text {eff }}^{l}=0.000013$ can be expected. Together with an improved determination of the mass of the $W$ boson to a precision of some $6 \mathrm{MeV}$ through a scan of the $W W$ production threshold and with the measurements obtained at high energy running of the collider this will allow many high precision tests of the Standard Model at the loop level. As an example, figure 12 shows the variation of the fit $\chi^{2}$ to the electroweak measurements as a function of $M_{H}$ for the present data and for the data expected at a linear collider. The mass of the Higgs boson can indirectly be constraint at a level of $5 \%$. Comparing this prediction with the direct measurement of $M_{H}$ consistency tests of the Standard Model can be performed at the quantum level or to measure free parameters in extensions of the Standard Model. This is 


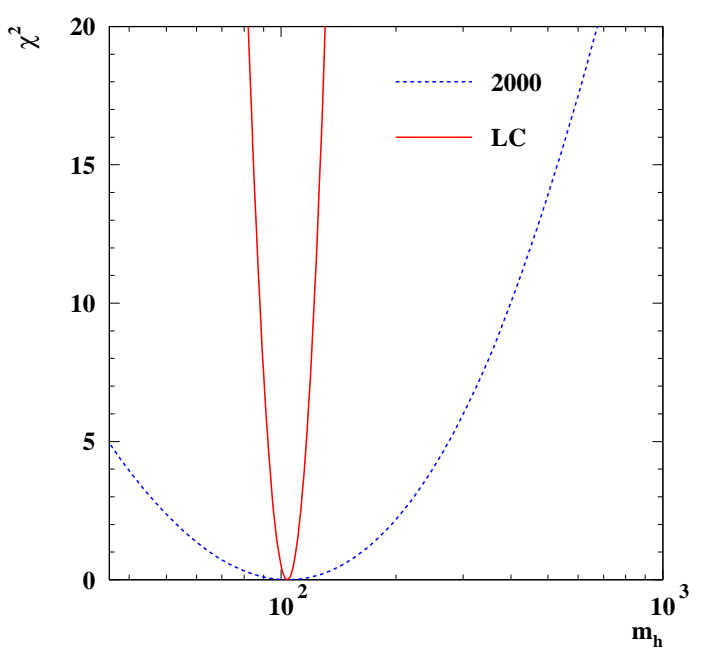

Figure 12. $\Delta \chi^{2}$ as a function of the Higgs boson mass for the electroweak precision data today (2000) and after GigaZ running (LC).

of particular importance if $M_{H}>200 \mathrm{GeV}$ in contradiction to the current electroweak measurements.

In summary, there is strong evidence for new phenomena at the TeV energy scale. Only the precision exploration at the linear collider will allow, together with the results obtained at the Large Hadron Collider, the understanding of the underlying physics and will open a new window beyond the centreof-mass energies reachable. Whatever scenario is realized in nature, the linear collider will add crucial information beyond the LHC. There is global consensus in the high energy physics community that the next accelerator based project needs to be an electronpositron linear collider with a centre-of-mass energy of at least $500 \mathrm{GeV}$.

\section{Electron-Positron Linear Colliders}

The feasibility of a linear collider has been successfully demonstrated by the operation of the SLAC Linear Collider, SLC. However, aiming at centre-of-mass energies at the $\mathrm{TeV}$ scale with luminosities of the order of $10^{34} \mathrm{~cm}^{-2} \mathrm{~s}^{-1}$ requires at least two orders of magnitude higher beam power and two orders of magnitude smaller beam sizes at the interaction point. Over the past decade, several groups worldwide have been pursuing different linear collider designs for the centre-ofmass energy range up to around one $\mathrm{TeV}$ as well as for the multi-TeV range. Excellent progress has been achieved at various test facilities worldwide in international collaborations on crucial aspects of the collider designs, At the Accelerator Test Facility at KEK 12, emittances within a factor two of the damping ring design have been achieved. At the Final Focus Test Beam at SLAC 13 demagnification of the beams has been proven; the measured spot sizes are well in agreement with the theoretically expected values. The commissioning and operation of the TESLA Test Facility at DESY 14 has demonstrated the feasibility of the TESLA technology. In the following, a short review of the different approaches is given.

\subsection{TeV range}

Three design studies are presently pursued: JLC 15, NLC 16 and TESLA 17, centred around KEK, SLAC and DESY, respectively. Details about the design, the status of development and the individual test facilities can be found in the above quoted references as well as in the status reports presented at LCWS2000 18, 19.20. A comprehensive summary of the present status can be found in the Snowmass Accelerator R\&D Report 21, here only a short discussion of the main features and differences of the three approaches will be given with emphasis on luminosity and energy reach.

One key parameter for performing the physics program at a collider is the centreof-mass energy achievable. The energy reach 
of a collider with a given linac length and a certain cavity filling factor is determined by the gradient achievable with the cavity technology chosen. For normalconducting cavities the maximum achievable gradient scales roughly proportional to the RF frequency used, for superconducting Niobium cavities, the fundamental limit today is around 55 $\mathrm{MV} / \mathrm{m}$.

The second key parameter for the physics program is the luminosity $\mathcal{L}$, given by

$$
\mathcal{L}=\frac{n_{b} N_{e}^{2} f_{\text {rep }}}{4 \pi \sigma_{x}^{*} \sigma_{y}^{*}} H_{D}
$$

where $n_{b}$ is the number of bunches per pulse containing $N_{e}$ particles, $f_{r e p}$ is the pulse repetition frequency, $\sigma_{x, y}^{*}$ the horizontal (vertical) beamsize at the interaction point, and $H_{D}$ the disruption enhancement factor. An important constraint on the choice of the parameters is the effect of beamstrahlung due to the emission of synchrotron radiation. The average fractional beam energy loss $\delta_{E}$ is proportional to $\frac{1}{\left(\sigma_{x}^{*}+\sigma_{y}^{*}\right)^{2}}$. Choosing a flat beam size $\left(\sigma_{x}^{*} \gg \sigma_{y}^{*}\right)$ at the interaction point, $\delta_{E}$ becomes independent of the vertical beam size and the luminosity can be increased by reducing $\sigma_{y}^{*}$ as much as possible. Since $\sigma_{y}^{*} \propto \sqrt{\epsilon_{y} \beta_{y}^{*}}$ this can be achieved by a small vertical beta function $\beta_{y}^{*}$ and a small normalised vertical emittance $\epsilon_{y}$ at the interaction point. The average beam power $P_{\text {beam }}=\sqrt{s} n_{b} N_{e} f_{\text {rep }}=\eta P_{A C}$ is obtained from the mains power $P_{A C}$ with an efficiency $\eta$. Equation (11) can then be rewritten as

$$
\mathcal{L} \propto \frac{\eta P_{A C}}{\sqrt{s}} \sqrt{\frac{\delta_{E}}{\epsilon_{y}}}
$$

High luminosity therefore requires high efficiency $\eta$ and high beam quality with low emittance $\epsilon_{y}$ and low emittance dilution $\Delta \epsilon / \epsilon \propto f_{R F}^{6}$, which is largely determined by the RF frequency $f_{R F}$ of the chosen technology.

The fundamental difference between the three designs is the choice of technology for the accelerating structures. The design of NLC is based on normalconducting cavities using $f_{R F}$ of $11.4 \mathrm{GHz}$ (X-band), for JLC two options, X-band or C-band $(5.7 \mathrm{GHz})$ are pursued. The TESLA concept, developed by the TESLA collaboration, is using superconducting cavities $(1.3 \mathrm{GHz})$. As an example for a linear collider facility, figure 13 shows the schematic layout of TESLA.

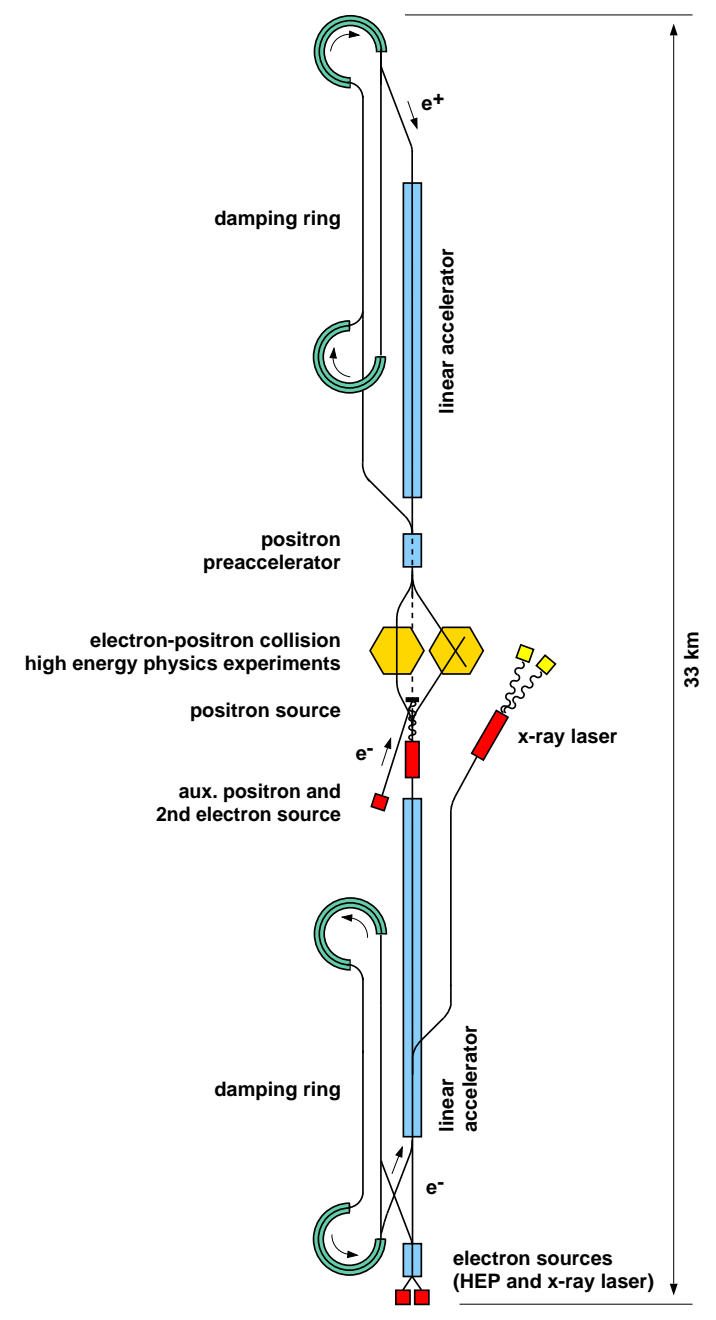

Figure 13. Schematic layout of TESLA

Table 2 compares some key parameters for the different technologies at $\sqrt{s}=500$ $\mathrm{GeV}$, like repetition rate $f_{\text {rep }}$ for bunch trains 


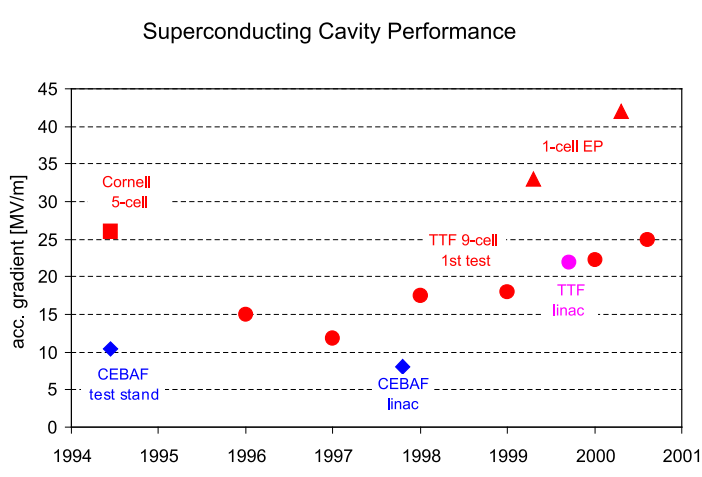

Figure 14. Evolution of superconducting cavity performance. The average gradient achieved with TESLA 9-cell cavities produced in industry (first test, no additional processing) is shown as dots.

with $N_{b}$ bunches, the time $\Delta T_{b}$ between bunches within a train which allows head on crossing of the bunches for TESLA but requires a crossing angle for the other designs. The design luminosity $\mathcal{L}$, beam power $P_{\text {beam }}$ and the required mains power $P_{A C}$ illustrate that for a given mains power the superconducting technology delivers higher luminosity. On the other hand the lower gradient $G_{a c c}$ requires a longer linac for the same centre-of-mass energy reach. As can be seen from table 2 the X-band machines call for a beam loaded (unloaded) gradient of some 50 (70) $\mathrm{MV} / \mathrm{m}$ for $\sqrt{s}$ of $500 \mathrm{GeV}$. Recently, it has been found that high gradient operation of normalconducting cavities results in surface damage of the structures. Intense $R \& D$ is going on in collaboration between SLAC, KEK and CERN in order to understand and resolve the problem. At present it seems that the onset of the damage depends on the structure length and the group velocity within the cavity 16 .

The TESLA design requires $23.5 \mathrm{MV} / \mathrm{m}$ for $\sqrt{s}=500 \mathrm{GeV}$, a gradient which is meanwhile routinely achieved for cavities fabricated in industry as illustrated in figure 14 .

Table 2 also contains the presently planned length of the facilities $17,16,22$ 23. An

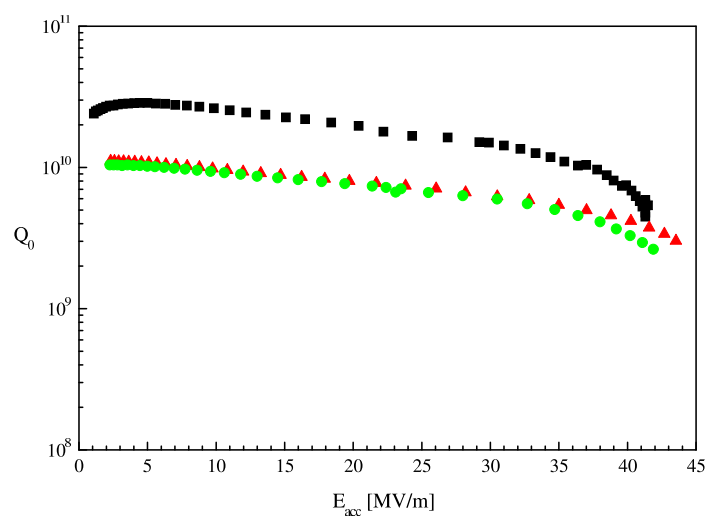

Figure 15. Excitation curves of three electropolished single-cell cavities. Gradients well above $35 \mathrm{MV} / \mathrm{m}$ are reached.

upgrade in energy up to around one $\mathrm{TeV}$ seems possible for all designs. In the NLC case, more cavities would be installed within the existing tunnel, in the JLC case, the tunnel length would have to be increased to house more cavities. In the TESLA case, a gradient of around $35 \mathrm{MV} / \mathrm{m}$ is needed to reach $\sqrt{s}$ of $800 \mathrm{GeV}$ within the present tunnel length. Higher energies would probably require an extension of the tunnel. Such gradients have repeatedly been reached in tests of single-cell cavities whose surfaces have been electropolished not only chemically treated. The result of this common effort from KEK, CERN, Saclay and DESY is shown in figure 15 .

In summary, all designs are very well advanced. The TESLA collaboration has presented a fully costed Technical Design Report in March 2001. The other collaborations are expected to provide such reports within the next years. The construction of a linear collider with at least $500 \mathrm{GeV}$ centre-of-mass energy, with upgrade potential to around one $\mathrm{TeV}$, could start soon.

\subsection{Multi-TeV Range}

To reach centre-of-mass energies beyond the TeV range, up to 3-5 TeV, a two beam accel- 
For Publisher's use

Table 2. Comparison of some crucial parameters at $500 \mathrm{GeV}$ for the different technologies under study, see text for details.

\begin{tabular}{|l|l|l|l|l|}
\hline & TESLA & NLC & JLC-X & JLC-C \\
\hline$f_{\text {rep }}[\mathrm{Hz}]$ & 5 & 120 & 150 & 100 \\
$N_{b}$ & 2820 & 190 & 190 & 142 \\
$\Delta T_{b}[\mathrm{~ns}]$ & 337 & 1.4 & 1.4 & 2.8 \\
bunch crossing & head on & angle & angle & angle \\
$N_{e} /$ bunch $\left[10^{10}\right]$ & 2 & .75 & 0.7 & 1.11 \\
\hline$\sigma_{x / y}^{*}[$ nm $]$ & $553 / 5$ & $245 / 2.7$ & $239 / 2.57$ & $318 / 4.3$ \\
$\delta_{E}[\%]$ & 3.2 & 4.7 & 5.3 & 3.9 \\
\hline $\mathcal{L}\left[10^{34} \mathrm{~cm}^{-2} s^{-1}\right]$ & 3.4 & 2 & 2.64 & 1.3 \\
\hline$P_{\text {beam }}[M W]$ & 22.6 & 13.2 & 17.6 & 12.6 \\
$P_{A C}($ linacs $)[M W]$ & 97 & 132 & 141 & 220 \\
\hline$G_{\text {acc }}[\mathrm{MV} / \mathrm{m}]$ & 23.5 & 48 & 50.2 & 36 \\
$L_{\text {tot }}[\mathrm{km}]$ & 33 & 30 & 16 & linac 19 \\
\hline
\end{tabular}

eration concept (CLIC) with very high accelerating fields is being developed at CERN 24. The schematic layout of that facility is shown in figure 16. It is optimised for $\sqrt{s}$ of $3 \mathrm{TeV}$, using high frequency $(30 \mathrm{GHz})$ normalconducting structures operating at very high accelerating fields $(150 \mathrm{MV} / \mathrm{m})$. The present design calls for bunch separations of .67 ns, a vertical spotsize of $1 \mathrm{~nm}$ and beamstrahlung $\delta_{E}$ of $30 \%$. For this promising concept a new test facility is under construction at CERN which should allow tests with full gradient starting in 2005.

\section{Realisation}

The new generation of high energy colliders most likely exceeds the resources of a country or even a region. There is general consensus that the realisation has to be done in an international, interregional framework. One such framework, the so called Global Accelerator Network (GAN), has been proposed to ICFA in March 2000. A short discussion of the principle considerations will be presented here, more details can be found in ref. 25.

The GAN is a global collaboration of laboratories and institutes in order to design, construct, commission, operate and main- tain a large accelerator facility. The model is based on the experience of large experimental collaborations, particularly in particle physics. Some key elements are listed below:

- it is not an international permanent institution, but an international project of limited duration;

- the facility would be the common property of the participating countries;

- there are well defined roles and obligations of all partners;

- partners contribute through components or subsystems;

- design, construction and testing of components is done in participating institutions;

- maintenance and running of the accelerator would be done to a large extent from the participating institutions.

The GAN would make best use of worldwide competence, ideas and resources, create a visible presence of activities in all participating countries and would, hopefully, make the site selection less controversial. 
For Publisher's use

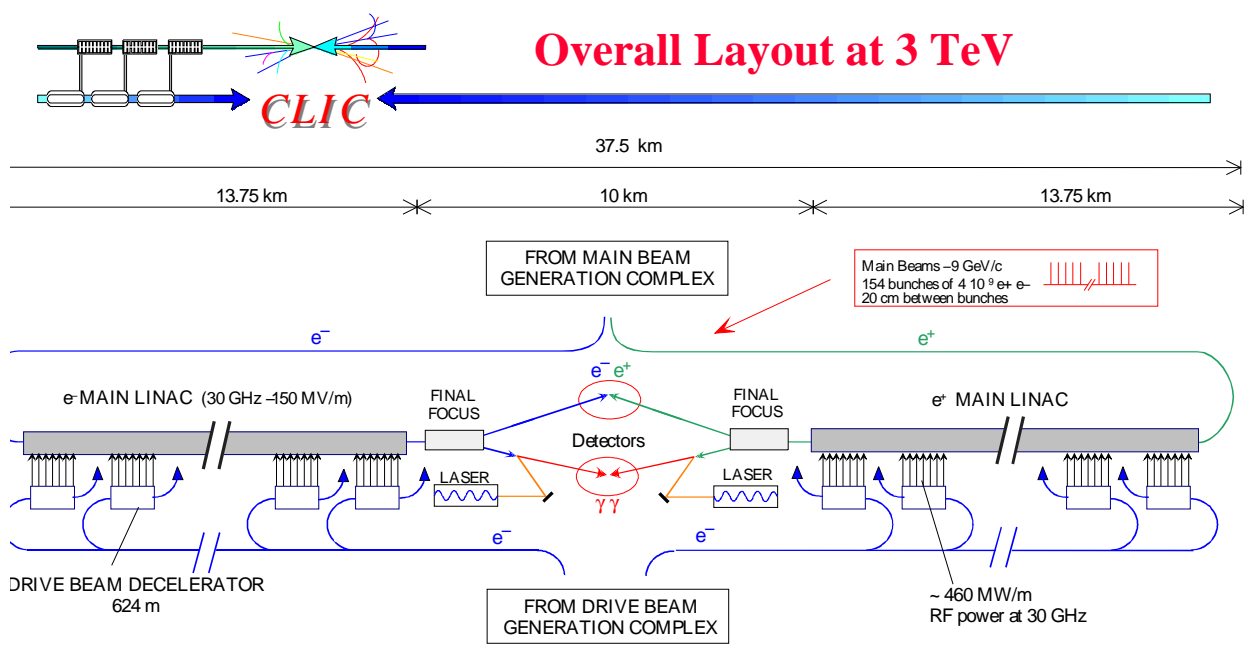

Figure 16. Overall layout of the CLIC complex for a centre-of-mass energy of $3 \mathrm{TeV}$.

ICFA has set up two working groups to study general considerations of implementing a GAN and to study the technical considerations and influence on the design and cost of the accelerator. The reports of these working groups can be found on the web 26. Their overall conclusion is that a GAN can be a feasible way to build and operate a new global accelerator, although many details still need to be clarified.

\section{Summary}

There is global consensus about the next accelerator based project in particle physics. It has to be an electron-positron linear collider with an initial energy reach of some $500 \mathrm{GeV}$ with the potential of an upgrade in centreof-mass energy. The physics case is excellent, only a few highlights could be presented here. There is also global consensus that concurrent operation with LHC is needed and fruitful. Therefore, a timely realisation is mandatory. The technical realisation of a linear collider is now feasible, several technologies are either ripe or will be ripe soon. A fast consensus in the community about the technology is called for having in mind a timely realisation as a global project with the highest possible luminosity and a clear upgrade potential beyond $500 \mathrm{GeV}$.

\section{Acknowledgments}

The author would like to express his gratitude to all people who have contributed to the studies of future electron-positron linear colliders from the machine design to physics and detector studies. Special thanks go to the organisers and their team for a very well organised, inspiring conference as well as for the competent technical help in preparing this presentation.

\section{References}

1. J.A. Aguilar-Saavedra et al, TESLA Technical Design Report, Part III, Physics at an $e^{+} e^{-}$Linear Collider, DESY 2001-011, ECFA 2001-209, hep$\mathrm{ph} / 0106315$.

2. T. Abe et al, Linear Collider Physics Resource Book for Snowmass 2001, BNL52627, CLNS 01/1729, FERMILABPub-01/058-E, LBNL-47813, SLAC-R- 
570, UCRL-ID-143810-DR, LC-REV2001-074-US, hep-ex/0106055-58

3. K. Abe et al, Particle Physics Experiments at JLC, KEK-Report 2001-11, hep-ph/0109166.

4. Proceedings of LCWS, Physics and Experiments with Future Linear Colliders, eds A. Para, H.E. Fisk, (AIP Conf. Proc., Vol 578, 2001).

5. Worldwide Study of the Physics and Detectors for Future $e^{+} e^{-}$Colliders http://lcwws.physics.yale.edu/lc

6. ACFA Joint Linear Collider Physics and Detector Working Group http://acfahep.kek.jp/

7. 2nd Joint ECFA/DESY Study on Physics and Detectors for a Linear Electron-Positron Collider http://www.desy.de/conferences/ecfadesy-lc98.html

8. A Study of the Physics and Detectors for Future Linear $e^{+} e^{-}$Colliders: American Activities

http://lcwws.physics.yale.edu/lc/america.htm

9. G. Alexander et al, TESLA Technical Design Report, Part IV, A Detector for TESLA, DESY 2001-011, ECFA 2001209.

10. H. N. Brown et al. [Muon g-2 Collaboration], Phys. Rev. Lett. 86 (2001) 2227

11. J. Drees, these proceedings

12. E.Hinode et al, eds., KEK Internal 954, 1995, eds J.Urakawa and M.Yoshioka, Proceedings of the SLAC/KEK Linear Collider Workshop on Damping Ring, KEK 92-6, 1992

13. The FFTB Collaboration: BINP (Novosibirsk/Protvino), DESY, FNAL, KEK, LAL(Orsay), MPI Munich, Rochester, and SLAC

14. Proposal for a TESLA Test Facility, DESY TESLA-93-01, 1992

15. KEK-Report 97-1, 1997.

16. Zeroth Order Design Report for the Next Linear Collider, SLAC Report
474, 1996. 2001 Report on the Next Linear Collider, Fermilab-Conf-01-075E, LBNL-47935, SLAC-R-571, UCRLID-144077

17. J. Andruszkow et al, TESLA Technical Design Report, Part II, The Accelerator, DESY 2001-011, ECFA 2001-209

18. O.Napoly, TESLA Linear Collider: Status Report, in ref $\Theta$

19. T.O. Raubenheimer, Progress in the Next Linear Collider Design, in ref 4

20. Y.H. Chin et al Status of JLC Accelerator Development, in ref 4

21. A. Chao et al, 2001 Snowmass Accelerator REBD Report, http://www.hep.an. gov/pvs/dpb/Snowmass.pdf

22. Y.H. Chin, private communication

23. H.Matsumoto, T.Shintake, private communication

24. I.Wilson, A Multi-TeV Compact $e^{+} e^{-}$ Linear Collider, in ref 4

25. F. Richard et al, TESLA Technical Design Report, Part I, Executive Summary, DESY 2001-011, ECFA 2001-209, hep$\mathrm{ph} / 0106314$.

26. http://www.fnal.gov/directorate/icfa/ icfa_tforce_reports.html 